\section{Allergopharma richtet sich neu aus}

Als Marktführer in der spezifischen Immuntherapie richtet sich das Unternehmen Allergopharma neu aus und wird sich zukünftig auf sein Kerngeschäft mit hochdosierten, hypoallergenen Produkten zur subkutanen Immuntherapie (SCIT) konzentrieren. Die erfolgreiche Entwicklung von Allergopharma beruht in erster Linie auf den SCITPräparaten Allergovit ${ }^{\circledR}$ und Acaroid ${ }^{\circledR}$. Auch langfristig wird die SCIT von großer Bedeutung sein für die spezifische Hyposensibilisierung, denn sie ist die bevorzugte Therapieform der Allergologen. Dies ist unter anderem darauf zurückzuführen, dass die SCIT dem Arzt eine enge Patientenführung ermöglicht und eine gute Compliance zur Folge hat. Ein weiterer Vorteil der SCIT liegt in ihrer nachgewiesenen langanhaltenden Wirksamkeit und ihrer guten Verträglichkeit sowie dem positiven Kosten-NutzenVerhältnis.

Allergopharma wird sein Kerngeschäft daher zukünftig auf hochdosierte, hypoallergene SCIT-Produkte fokussieren und diese kontinuierlich weiterentwickeln. So hat zum Beispiel Allergovit ${ }^{\circledR}$ Gräser- und Getreidepollen im August 2010 als erstes und einziges hypoallergenes SCITPräparat vom Paul-Ehrlich-Institut die Genehmigung zur Aufnahme des Langzeit- und Disease-Modifying-Effektes in die Fach- und Gebrauchsinformation erhalten. Für Acaroid ${ }^{\circledR}$ konnte in einer aktuellen Studie für Kinder mit Asthma bronchiale aufgrund einer Hausstaubmilbenallergie ein steroidsparender Effekt der SCIT nachgewiesen werden.

Die Entwicklung der hochdosierten Sublingualpräparate der dritten Generation, zu denen auch AllerSlit ${ }^{\circledR}$ forte gehört, entspricht dagegen nicht den Erwartungen des Unternehmens. Aus diesem Grund hat Allergopharma den Vertrieb von AllerSlit ${ }^{\circledR}$ forte für neue Patienten zum 1. Oktober 2010 eingestellt. Für Patienten, die sich bereits in der Therapie befinden, wird die weitere Versorgung selbstverständlich sichergestellt sein.

Im Bereich der hypoallergenen sowie hochdosierten SCIT wird Allergopharma weiterhin eine intensive Forschung betreiben und innovative Produkte, wie zum Beispiel biotechnologisch hergestellte Allergene, entwickeln.

Nach Informationen von Allergopharma, Reinbek

\section{Ein Quantensprung in die Zukunft der Hyposensibilisierung}

m nächsten Jahr feiert die spezifische

Immuntherapie ihren 100. Geburtstag, in dieser Zeitspanne hat sich viel getan. Ein entscheidender Schritt war die Entwicklung von hypoallergenen und hochdosierten Präparaten wie Allergovit ${ }^{\circledR}$. PD Dr. Randolf Brehler aus Münster rief Studiendaten zu den Langzeiteffekten aus dem Jahr 2009 in Erinnerung: Drei Jahre nach Ende einer Therapie mit Allergovit ${ }^{\circledR}$ lag der Symptom-Medikations-Score von rund 30 Patienten, die aufgrund einer Gräserpollenallergie hyposensibilisiert worden waren, ganze $81,4 \%(p<0,005)$ unter dem der Kontrollgruppe, die keine subkutane Immuntherapie erhalten hatte. „Es ist diese Studie, die auch dazu geführt hat, dass der Langzeiteffekt für dieses Präparat inzwischen im Beipackzettel dokumentiert ist", sagte Brehler. Eine Subanalyse zeigte, dass die anhaltende Verbesserung alle Bereiche der allergischen Erkrankung betraf: Für Augensymptome betrug der Unterschied der Symptomscores zwischen der Kontrollund der Verumgruppe $75 \%$, für Nasensymptome waren es $68 \%$ und für asthmatische Beschwerden 93\%. Auch hinsichtlich der Lebensqualität profitierten die behandelten Patienten von der Immuntherapie. Für Kinder wurden eine über zwölf Jahre anhaltende Langzeitwirkung und krankheitsmodifizierende Effekte bereits 2006 nachgewiesen.

Die neuesten Entwicklungen sind noch nicht auf dem Markt: Sie gehen in Richtung rekombinanter Allergene. PD Dr. Oliver Pfaar aus Wiesbaden erklärte ihre Herstellung: Die in den Zellkernen einer Allergenquelle enthaltene genetische Information (DNA) für ein allergenes Protein wird in Messenger-RNA (mRNA) umgesetzt. Die mRNA wird isoliert und dient als Vorlage für die Übersetzung in komplementäre DNA (cDNA) mithilfe des Enzyms Reverse Transkriptase. Über ein Virusplasmid wird die cDNA auf Wirtzellen (z.B. Bakterien, Hefen) übertragen und in deren genetische Information eingebaut. Die infizierten Wirtszellen sind dann in der Lage, das gewünschte Allergen zu

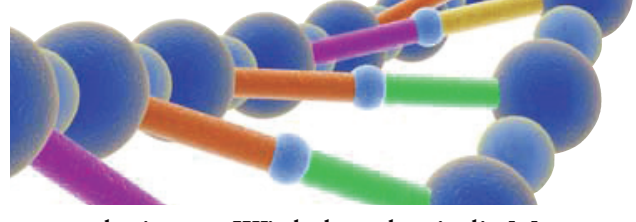

produzieren. „Wir haben damit die Möglichkeit, die Präparate so zu designen, wie wir sie haben wollen", freute sich Pfaar. Im Endprodukt seien dann nur noch die je nach Sensibilisierungsmuster der Patienten relevanten Allergene enthalten, durch die hohe Reinheit sei die Gefahr für Neusensibilisierungen reduziert und die rekombinanten Allergene könnten in vitro in unbegrenzter Menge hergestellt werden - für Pfaar ein Quantensprung.

Erste Erfahrungen mit der Sicherheit und Wirksamkeit von rekombinanten Allergenen gibt es schon. Eine Dosisfindungsstudie für einen äquimolaren Cocktail der fünf Phleum-pratense-Hauptallergene $\mathrm{Phl} \mathrm{p} \mathrm{1,} \mathrm{2,} \mathrm{5a,} 5 \mathrm{~b}$ und 6 verlief ohne schwerwiegende Reaktionen trotz einer Auftitration bis zu $120 \mu \mathrm{g}$. Die hohe Wirksamkeit des rPhleum in der ersten Saison bestätigte eine Phase-II-Studie. Weiter ist man bei der Birke: Alle mit der rekombinanten Bet-v-1-Faltungsvariante (rBet v 1-FV) behandelten Patienten erreichten die Erhaltungsdosis von $80 \mu \mathrm{g}$, ohne dass schwerwiegende Reaktionen auftraten. Im Vergleich zu einem Birkenstandardpräparat war das rekombinante in der Wirksamkeit in der ersten Saison sogar überlegen. In einer Phase-III-Studie wurde eine frühe und starke Induktion von spezifischem $\operatorname{IgG}_{4}$ festgestellt.

Prof. Dr. Ulrich Wahn aus Berlin fasste zusammen: „Die Immuntherapie ist sexy und innovativ!" Sie sei ohne Frage evidenzbasiert. Im Gegensatz zu allen anderen Pharmakotherapien beweise sie Langzeiteffekte und ein krankheitsveränderndes Potenzial. Überdies zeige sie bereits in der ersten Pollensaison eine den symptomatischen Therapien vergleichbare bzw. überlegene Wirksamkeit. we

Industriesymposium „Hochdosis-Immuntherapie: Voraussetzungen für den Erfolg“ im Rahmen des 5. Deutschen Allergiekongresses. Hannover, 10. September 2010. Veranstalter: Allergopharma, Reinbek 